Annales Academiæ Scientiarum Fennicæ

Mathematica

Volumen 40, 2015, 227-234

\title{
ROTATION NUMBERS AND SYMBOLIC DYNAMICS
}

\section{David Bowman, Ross Flek and Greg Markowsky}

\author{
University of Adelaide, School of Mathematical Sciences \\ Adelaide SA 5005, Australia; david.bowman@adelaide.edu.au \\ The New School, Eugene Lang College For Liberal Arts, Interdisciplinary Science Department \\ New York, NY 10011, U.S.A.; flekr@newschool.edu \\ Monash University, School of Mathematical Sciences \\ Victoria 3800, Australia; gmarkowsky@gmail.com
}

\begin{abstract}
A degree $c$ rotation set in $[0,1]$ is an ordered set $\left\{t_{1}, \ldots, t_{q}\right\}$ such that there is a positive integer $p$ such that $c t_{i}(\bmod 1)=t_{i+p(\bmod q)}$ for $i=1, \ldots, q$. The rotation number of the set is defined to be $\frac{p}{q}$. Goldberg has shown that for any rational number $\frac{p}{q} \in(0,1)$ there is a unique quadratic rotation set with rotation number $\frac{p}{q}$. This result was used by Goldberg and Milnor to study Julia sets of quadratic polynomials [8].

In this work, we provide an alternate proof of Goldberg's result which employs symbolic dynamics. We also deduce a number of additional results from our method, including a characterization of the values of the elements of the rotation sets.
\end{abstract}

\section{Introduction}

Let $f$ be a complex polynomial in one variable and let $z_{0}$ be a fixed point of $f$. Then $z_{0} \in K(f)$, the filled-in Julia set of $f$. Define the rational type $T_{0}$ of $z_{0}$ to be the set of all rational angles $\theta$, such that the external dynamic ray of angle $\theta$ lands at $z_{0}$. The fixed-point portrait of $f$, denoted by $P(f)$, is the set of all such $T_{j}$, where $T_{j}$ is the rational type of the fixed point $z_{j}$ of $f$.

The above terminology was developed by Goldberg and Milnor in [8] as a way of classifying polynomials of degree two or higher with connected Julia set. Their main theorem establishes when a given fixed-point portrait is that of a critically preperiodic polynomial, that is, a polynomial whose critical points are all strictly preperiodic.

Central to the results of Goldberg and Milnor is the notion of a rotation set (see Section 2), which Goldberg defines in [7]. The main theorem of Goldberg's paper establishes that a rotation set is uniquely determined by its rotation number and deployment sequence. The quadratic case of this theorem, relating to rotation sets under the doubling map, takes a particularly elegant form: for rational number $\frac{p}{q} \in(0,1)$ there is a unique quadratic rotation set with rotation number $\frac{p}{q}$.

Goldberg's proof relies heavily on geometric arguments. It is noteworthy, however, that Goldberg's result can be stated entirely without reference to complex dynamics, or any sort of geometric interpretation. As such, it is logical to seek an alternate proof which dispenses with this additional machinery. Our goal in this note is to provide a proof of Goldberg's main theorem on quadratic rotation sets using purely symbolic arguments. We also show that such an approach can simplify some of the auxiliary results in Goldberg and Milnor's paper in the degree two case.

doi:10.5186/aasfm.2015.4015

2010 Mathematics Subject Classification: Primary 37E45, 37E10, 37E15.

Key words: Symbolic dynamics, rotation sets, complex dynamics, doubling map, combinatorial dynamics. 


\section{The doubling map, rotation sets and the main theorem}

Let $t \in \mathbf{R} / \mathbf{Z}$. Define the doubling map on $\mathbf{R} / \mathbf{Z}$ as $D(t)=2 t(\bmod 1)$. Let $t \in \mathbf{R} / \mathbf{Z}$; we define the (forward) orbit of $t$ under $D$ as $\operatorname{Orb}_{D}(t):=\bigcup_{n \geq 0} D^{\circ n}(t)$. It is easy to see that $\operatorname{Orb}_{D}(t)$ is finite if and only if $t$ is rational. We will say that a point $t_{0}$ is periodic if there is a positive integer $q$ such that $D^{\circ q}\left(t_{0}\right)=t_{0}$, and if $t_{0}$ is periodic we will let the period of $t_{0}$ be the smallest such $q$, and say that $t_{0}$ is $q$-periodic. The orbit of such a point consists of $q$ periodic points, each of period $q$, and is called a cycle of period $q$. Note that $D(t)$ has one fixed point at $t=0$, or, equivalently, $t=1$. The following proposition is well known and its proofs and applications are plentiful, for example, see $[3,5,9]$.

Proposition 1. Let $a, b \in \mathbf{Z}^{+}$with $a<b$. Then $t_{0}=\frac{a}{b}$ is periodic if and only if $b$ is odd.

Note that if $t_{0}=\frac{a}{2^{n} r}$ where $r$ is odd then $D^{\circ n}\left(t_{0}\right)$ is periodic. Let $t_{0}$ be $q$-periodic. Define the ordered orbit of $t_{0}$, denoted by $\overrightarrow{\mathrm{Orb}}_{D}\left(t_{0}\right)$, as the set of all elements of $\operatorname{Orb}_{D}\left(t_{0}\right)$ written in increasing order, that is, $\overrightarrow{\mathrm{Orb}}_{D}\left(t_{0}\right)=\left\{t_{1}, t_{2}, t_{3}, \ldots, t_{q}\right\}$ where each $t_{i} \in \operatorname{Orb}_{D}(t)$ and $t_{1}<t_{2}<t_{3}<\cdots<t_{q}$. We will say that $\overrightarrow{\operatorname{Orb}}_{D}\left(t_{0}\right)=$ $\left\{t_{1}, t_{2}, t_{3}, \ldots, t_{q}\right\}$ is a rotation set if there exists a fixed positive integer $p<q$ such that for each $t_{i}, D\left(t_{i}\right)=t_{(i+p) \bmod q}$. We will refer to the rational number $\frac{p}{q}$ as the rotation number of the rotation set. To illustrate, let us note that if we take $t_{0}=\frac{1}{5}$, we have

$$
\operatorname{Orb}_{D}\left(\frac{1}{5}\right)=\left\{\frac{1}{5}, \frac{2}{5}, \frac{4}{5}, \frac{3}{5}\right\}, \quad \overrightarrow{\mathrm{Orb}}_{D}\left(\frac{1}{5}\right)=\left\{\frac{1}{5}, \frac{2}{5}, \frac{3}{5}, \frac{4}{5}\right\} .
$$

Since $D\left(t_{1}\right)=D\left(\frac{1}{5}\right)=\frac{2}{5}=t_{2}$ while $D\left(t_{2}\right)=D\left(\frac{2}{5}\right)=\frac{4}{5}=t_{4}$, we see that $\overrightarrow{\operatorname{Orb}}_{D}\left(\frac{1}{5}\right)$ is not a rotation set. As a matter of fact, the reader may want to verify that there exists no rotation set whose elements have denominator 5 . On the other hand, let us take $t_{0}=\frac{5}{31}$. Then

(2) $\operatorname{Orb}_{D}\left(\frac{5}{31}\right)=\left\{\frac{5}{31}, \frac{10}{31}, \frac{20}{31}, \frac{9}{31}, \frac{18}{31}\right\}, \quad \overrightarrow{\operatorname{Orb}}_{D}\left(\frac{5}{31}\right)=\left\{\frac{5}{31}, \frac{9}{31}, \frac{10}{31}, \frac{18}{31}, \frac{20}{31}\right\}$.

It may now easily be checked that $\overrightarrow{\mathrm{Orb}}_{D}\left(\frac{5}{31}\right)$ is a rotation set with rotation number $\frac{2}{5}$. As another example, note that $\overrightarrow{\mathrm{Orb}}_{D}\left(\frac{1}{31}\right)$ is a rotation set with rotation number $\frac{1}{5}$. It is a worthwhile and somewhat illuminating exercise to find the rotation sets for rotation numbers $\frac{3}{5}$ and $\frac{4}{5}$. Clearly, then, some rational values give rise to rotation sets, while others do not. A consequence of our work below is a characterization, in terms of binary expansions, of which values yield rotation sets. For $t_{j}, t_{k} \in\left\{t_{1}, t_{2}, t_{3}, \ldots, t_{q}\right\}$, we define $\gamma\left(t_{j}, t_{k}\right)=\min _{m>0}(j+m) \bmod q=k$ to be the forward distance between indices of $t_{j}, t_{k}$. The following proposition helps justify the terms rotation set and rotation number.

Proposition 2. Let $t_{0}$ be q-periodic. The following are equivalent.

(i) $\overrightarrow{\mathrm{Orb}}_{D}\left(t_{0}\right)=\left\{t_{1}, t_{2}, t_{3}, \ldots, t_{q}\right\}$ is a rotation set.

(ii) For any $t_{j}, t_{k} \in\left\{t_{1}, t_{2}, t_{3}, \ldots, t_{q}\right\}, \gamma\left(D\left(t_{j}\right), D\left(t_{k}\right)\right)=\gamma\left(t_{j}, t_{k}\right)$.

We omit the straightforward proof. The following is a key characterization of rotation sets. 
Proposition 3. Let $t_{0}$ be q-periodic. Then $\overrightarrow{\mathrm{Orb}}_{D}\left(t_{0}\right)=\left\{t_{1}, t_{2}, t_{3}, \ldots, t_{q}\right\}$ is a rotation set if and only if $t_{q}-t_{1}<\frac{1}{2}$.

We will give a simple proof of this result in the following section. We are now ready to state our main theorem, which was given as a special case of Theorem 7 in [7].

Theorem 1. Let $p, q \in \mathbf{Z}^{+}$with $p$ and $q$ relatively prime, and $p<q$. Then there exists a unique rotation set whose rotation number is $\frac{p}{q}$.

The remainder of this paper will be devoted to proving this theorem and related discussions. This result allows us to refer to such a set as $\operatorname{Rot}_{2}\left(\frac{p}{q}\right)$. We will call this the $\frac{p}{q}$-rotation set of degree 2 .

Interest in this result has arisen at least partially due to its importance in complex dynamics. In [8], Goldberg and Milnor used rotation sets to study fixed points of quadratic polynomials. Given a quadratic polynomial with connected Julia set, a fixed point of $f$ is rationally visible if it is the landing point of an external ray with rational angle. Goldberg and Milnor proved that a rationally visible fixed point is either hit by the 0-ray, or by the set of all rays whose angles are the elements of a quadratic rotation set. For example, the Julia set of the polynomial $e^{(2 \pi i) \frac{2}{5}} z+z^{2}$ is shown in Figure 1 below. The fixed point 0 is rationally visible, and the angles of the rays landing at 0 are the elements of the rotation set given in (2).

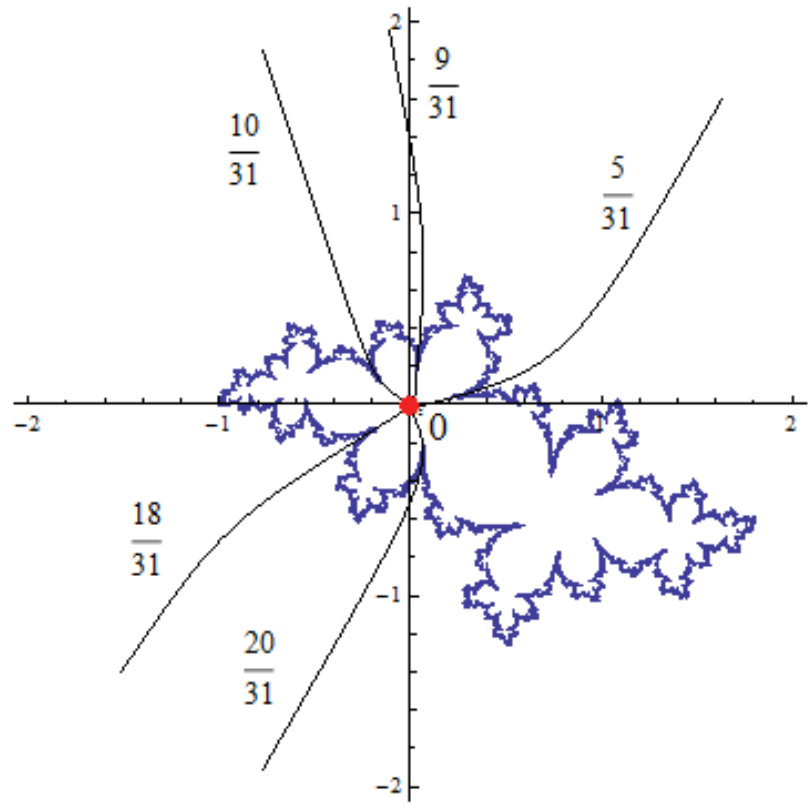

Figure 1. The Julia set of $e^{(2 \pi i) \frac{2}{5}} z+z^{2}$. Note the angles of the external rays landing at the origin are the elements of $\operatorname{Rot}_{2}\left(\frac{2}{5}\right)$. Letting $R_{1}$ be the ray of angle $\frac{5}{31}, R_{2}$ the ray of angle $\frac{9}{31}$ and so on, every application of the complex map rotates the five external rays, mapping each $R_{i}$ to $R_{i+2}$; after five iterations, all five external rays return to their original position.

\section{Binary string interpretations}

We begin with the proof of Proposition 3.

Proof of Proposition 3. Suppose first that $t_{q} \geq t_{1}+1 / 2$. Then $t_{q} \geq 1 / 2$ and $t_{1} \leq 1 / 2$, so that $D\left(t_{1}\right)=2 t_{1} \leq 2 t_{q}-1=D\left(t_{q}\right)$. This violates (ii) from Proposition 
2 , since $\gamma\left(t_{q}, t_{1}\right)=1$, and the only way that we could have $\gamma\left(D\left(t_{q}\right), D\left(t_{1}\right)\right)=1$ with $D\left(t_{q}\right) \geq D\left(t_{1}\right)$ is if $D\left(t_{q}\right)=t_{q}, D\left(t_{1}\right)=t_{1}$. Thus, $\left\{t_{1}, t_{2}, t_{3}, \ldots, t_{q}\right\}$ is not a rotation set. Conversely, suppose $t_{q}<t_{1}+1 / 2$. For $t^{\prime}, t^{\prime \prime} \in[0,1)$, let $\mathcal{I}\left(t^{\prime}, t^{\prime \prime}\right)$ be $\left[t^{\prime}, t^{\prime \prime}\right]$ if $t^{\prime} \leq t^{\prime \prime}$ and $\left\{t \in[0,1) ; t \geq t^{\prime}\right.$ or $\left.t \leq t^{\prime \prime}\right\}$ if $t^{\prime}>t^{\prime \prime}$. Let $t_{j}, t_{k} \in\left\{t_{1}, t_{2}, t_{3}, \ldots, t_{q}\right\}$, and set $m=$ $\gamma\left(t_{j}, t_{k}\right)$. Then $A=\left\{t_{j}, t_{(j+1) \bmod q}, \ldots, t_{(j+m-1) \bmod q}, t_{k}\right\}$ is a cyclicly ordered set, and $\mathcal{I}\left(t_{j}, t_{k}\right) \cap\left\{t_{1}, t_{2}, t_{3}, \ldots, t_{q}\right\}=A$. It is clear that $D(A)=\left\{D\left(t_{j}\right), D\left(t_{(j+1) \bmod q}\right), \ldots\right.$, $\left.D\left(t_{(j+m-1) \bmod q}\right), D\left(t_{k}\right)\right\}$ is cyclicly ordered as well. Furthermore, since $t_{q}-t_{1}<1 / 2$, $D$ is injective on $\left[t_{1}, t_{q}\right]$, so that $\mathcal{I}\left(D\left(t_{j}\right), D\left(t_{k}\right)\right) \cap\left\{t_{1}, t_{2}, t_{3}, \ldots, t_{q}\right\}=D(A)$. It follows that $\gamma\left(D\left(t_{j}\right), D\left(t_{k}\right)\right)=m=\gamma\left(t_{j}, t_{k}\right)$, and thus $\left\{t_{1}, t_{2}, t_{3}, \ldots, t_{q}\right\}$ is a rotation set by (ii) of Proposition 2.

We begin our efforts towards the goal of proving Theorem 1 by recalling that periodic points under $D$ can be expressed as rational numbers with odd denominators. It will be convenient for our purposes to express fractions as repeating binary decimals. The following well known proposition characterizes the binary expansions of rational numbers with odd denominators. Varying versions, proofs and examples of this proposition may be found in several symbolic dynamics articles, such as [1] or [2]. The proof is included to further motivate the subsequent discussion.

Proposition 4. Let $\frac{a}{b}$ be a rational number in $[0,1)$ with $G C D(a, b)=1$. Then the binary expansion of $\frac{a}{b}$ is of the form .SSSSS..., where $S$ is a finite block of 0 and 1 's if and only if $b$ is odd.

Proof. Consider a rational number of the form $\frac{c}{d}=. S S S S S \ldots$. with $q$ denoting the length of the string $S$. Then the binary expansion of $\left(2^{q}-1\right) \frac{c}{d}$ is $S$. We conclude that $\frac{a}{b}$ being of the form.$S S S S S \ldots$ is equivalent to being equal to a rational number with denominator equal to $2^{q}-1$ for some integer $q$, which is equivalent to $b$ dividing $2^{q}-1$. It is clear that this can only occur if $b$ is odd. To show sufficiency, we must show that any arbitrary odd $b$ divides $2^{q}-1$, for some $q$. To see that this must be so, note that we can find $m>n$ such that $2^{m} \equiv 2^{n}(\bmod b) .2$ is always invertible in the ring $\mathbf{Z} / b \mathbf{Z}$ when $b$ is odd, so we can conclude that $2^{m-n} \equiv 1(\bmod b)$, and thus $b \mid\left(2^{m-n}-1\right)$.

This proposition shows that we may reformulate the discussion of rotation sets in terms of binary strings. It should also be noted that, in the symbolic dynamics context, the binary expansion of points in $[0,1)$ defines a factor map from $\Sigma_{2}^{*}$ onto the circle. Let $\Sigma_{2}^{*}$ be the space of repeating one-sided infinite binary sequences. That is, every $X \in \Sigma_{2}^{*}$ can be realized as the concatenation $S S S S S \ldots$, where $S$ is a finite block of 0 and 1 's. We will write $S=1^{a_{1}} 0^{b_{1}} 1^{a_{2}} 0^{b_{2}} \ldots 1^{a_{k}} 0^{b_{k}}$ to signify the string formed by $a_{1} 1$ 's followed by $b_{1} 0$ 's, followed by $a_{2} 1$ 's, etc. Let $\sigma$ be the usual left shift map on $\Sigma_{2}^{*}$, given by $\sigma\left(x_{1} x_{2} x_{3} \ldots\right)=x_{2} x_{3} x_{4} \ldots$ To every $X \in \Sigma_{2}^{*}$ we will associate the finite string $S_{X}=x_{1} x_{2} x_{3} \ldots x_{q}$ of length $q$, where $S_{X}$ is the shortest possible repeating block of $X$. Conversely, given a finite binary string $S$, let $X_{S}$ be the periodic binary sequence given by the concatenation $S S S \ldots$. Let $S$ and $T$ be two different strings of length $q$. Then we will say that $S$ is cyclically equivalent to $T$ if $S$ is a cyclic permutation of $T$, or equivalently if there exists a $p<q$ such that $\sigma^{p}\left(X_{T}\right)=X_{S}$. In this case we will write $S \sim T$, and we will denote the equivalence class of $S$ under this relation by $[S]$. We will call the corresponding sequences $X_{S}$ and $X_{T}$ cyclically equivalent as well, and similarly denote the equivalence class of such sequences $\left[X_{S}\right]$ or $\left[X_{T}\right]$. Let $\hat{\Sigma}_{2}^{*}$ denote the space of equivalence classes in $\Sigma_{2}^{*}$. 
For any finite string $S$, set $M(S)=. S S S \ldots$, that is, $M(S)$ is the rational number whose binary expansion consists of a decimal point following by infinitely repeated copies of $S$. For example, $M(101)=.101101101 \ldots=\frac{5}{7}$. In general, if the length of $S$ is $q$ then $M(S)$ can be expressed as a fraction with denominator $2^{q}-1$. Likewise, for $X \in \Sigma_{2}^{*}$ let $M(X)=M\left(S_{X}\right)$. Given $[X] \in \hat{\Sigma}_{2}^{*}$, let $X_{\max }$ denote the maximal element of $[X]$, that is, the member of $[X]$ such that $M\left(X_{\max }\right) \geq M(Y)$ for all $Y \in[X]$. Let $X_{\min }$ be the minimal element of $[X]$, defined analogously. Let the width of the class $[X]$ be defined to be $d\left(X_{\max }, X_{\min }\right)=M\left(X_{\max }\right)-M\left(X_{\min }\right)$. We should note here that we are implicitly appealing to the fact that the lexicographic ordering of sequences in $\Sigma_{2}^{*}$ corresponds to geometric ordering on $[0,1)$. We will call $[X]$ admissible if $d\left(X_{\max }, X_{\min }\right)<\frac{1}{2}$. Let $C_{q}^{p}$ be the set of all equivalence classes of the form $\left[X_{S}\right]$, where $S$ is a binary string of length $q$ containing $p 1$ 's. Proposition 3 reveals the following to be simply a restatement of Theorem 1 in the language of binary strings.

Theorem 2. Let $\frac{p}{q} \in[0,1]$ be a rational number. Then $C_{q}^{p}$ contains a unique admissible equivalence class $[X]$.

Note that it is not assumed here that $G C D(p, q)=1$, although it will be shown in the next theorem that it is enough to consider only that case. We will see in what follows that our proof gives somewhat more information about the admissible equivalence class than is stated in Theorem 2. Call two strings $S$ and $T$ converses of each other if they are each equal to the other with its digits reversed; in other words, S read left to right is equal to T read right to left. So, for example, 01001 and 10010 are converses of each other. A string which is its own converse will be called a palindrome. An example of this would be 10101. For a finite binary string $S$ let the density of $S$ be defined to be $\frac{p}{q}$, where $q$ is the length of $S$ and $p$ is the number of 1's in $S$. The following is immediate from the methods we will use to prove Theorem 2.

Theorem 3. Let $\frac{p}{q} \in(0,1)$. Let $[X]$ be the unique admissible equivalence class in $C_{q}^{p}$ which is guaranteed by Theorem 2. Suppose that $X_{\max }=\overline{x_{1} x_{2} \ldots x_{q}}$ and $X_{\min }=\overline{y_{1} y_{2} \ldots y_{q}}$. Then the following hold.

i) $x_{1}=1, x_{q}=0, y_{1}=0, y_{q}=1$, and $x_{i}=y_{i}$ for $2 \leq i \leq q-1$.

ii) $S_{X_{\max }}$ and $S_{X_{\min }}$ are converses, or equivalently, $x_{2} x_{3} \ldots x_{(q-1)}$ is a palindrome.

iii) If $\left[X_{S}\right] \in C_{n q}^{n p}$ for any positive integer $n$ is admissible, then $S=T^{n}$ with $\left[X_{T}\right]$ the admissible class in $C_{q}^{p}$; that is, $S$ is the concatenation of $n$ copies of a string of length $q$ containing $p$ 1's corresponding to the unique admissible element in $C_{q}^{p}$. Thus, for any rational $\frac{p}{q} \in[0,1]$, with $p$ and $q$ relatively prime, there is a unique admissible equivalence class in $\hat{\Sigma}_{2}^{*}$ with density $\frac{p}{q}$, and this can be found in $C_{q}^{p}$.

This theorem allows us to prove information about the geometry of a rotation set. Making the obvious identification between $\mathbf{R} / \mathbf{Z}$ and the circle $\mathbf{T}$, of circumference 1 , we see Proposition 3 guarantees that a rotation set, $\operatorname{Rot}_{2}(p / q)$, is located entirely in an arc of length less than a half. By combining part $(i)$ and (ii) of this theorem, we see the shortest arc containing $\operatorname{Rot}_{2}(p / q)$ is of length $\frac{1}{2}-\frac{1}{2^{q}-1}$. Indeed, in binary form $S_{X_{\max }}=1 P 0$ and $S_{X_{\min }}=0 P 1$, for where P a palindrome of length $q-2$, giving $M\left(X_{\max }\right)-M\left(X_{\min }\right)=.01^{q-1} 01^{q-1} \ldots=\frac{1}{2}-\frac{1}{2^{q}-1}$. 
Furthermore, a rotation set partitions $\mathbf{T}$ into a disjoint series of arcs; namely the connected components of $\mathbf{T} \backslash \operatorname{Rot}_{2}(p / q)$. Indeed, given two adjacent elements, $t_{i}, t_{i+1} \in \operatorname{Rot}_{2}(p / q)$, with indices reduced $\bmod q$, these arcs are just the intervals $V_{i}:=\mathcal{I}\left(t_{i}, t_{i+1}\right)$. The length of $V_{i}$ is hence given by $l\left(V_{i}\right)=\left(t_{i+1}-t_{i}\right)$ for $i=$ $1, \ldots, q-1$, and $l\left(V_{q}\right)=1+t_{1}-t_{q}$. We then have

Proposition 5. The arc of greatest length is $V_{q}$, and its forward image under doubling, $V_{p}$, is the arc of shortest length. Moreover, the length of $V_{i}$ is given by

$$
l\left(V_{i}\right)=\frac{2^{n-1}}{2^{q}-1} \bmod 1,
$$

where $i=n p(\bmod q)$ and $1 \leq n \leq q$.

Proof. Since $M\left(X_{\max }\right)-M\left(X_{\min }\right)=.01^{q-1} 01^{q-1} \ldots$, it follows $V_{q}$ has the complementary length of $.10^{q-1} 10^{q-1} \ldots$. Since any $t_{i} \in \operatorname{Rot}_{2}(p / q)$ can be written of the form $t_{i}=D^{\circ n}\left(t_{q}\right)$ for some $n$, it follows $l\left(V_{i}\right)=2^{n} l\left(V_{q}\right) \bmod 1$. The lengths of the $V_{i}$ 's are thus the elements of $\operatorname{Rot}_{2}(1 / q)$, the largest of which is $l\left(V_{q}\right)=.10^{q-1} 10^{q-1} \ldots$ and the smallest of which is its image under doubling, $l\left(V_{p}\right)=.0^{q-1} 10^{q-1} 1 \ldots$

We recall that Goldberg and Milnor [8] use rotation sets to study fixed points of quadratic polynomials. Given a polynomial $f(z)=z^{2}+c$ with connected Julia set, a fixed point of $f$ is rationally visible if it is the landing point of an external ray with rational angle. Goldberg and Milnor prove a rationally visible fixed point is either hit by the 0-ray, or by all of the elements of a rotation set $\operatorname{Rot}_{2}\left(\frac{p}{q}\right)$. These rays partition $\mathbf{C}$ into sectors. The sectors have angular width equal to the lengths of the $V_{i}$ given above. Furthermore, Goldberg and Milnor proved that the sector of smallest angular width contains the critical value $c$ and the rays bounding this sector are the only two parameter rational rays to land at $c$. This smallest sector in our notation is $V_{p}=\left(t_{p}, t_{p+1}\right)$. The critical point, 0 , is contained in sector of largest angular width, corresponding in our notation to $V_{q}=\left(t_{q}, t_{1}\right)$.

\section{Proofs of Theorems 2 and 3}

The cases $\frac{p}{q}=0$ and $\frac{p}{q}=1$ are trivial, so we will assume $\frac{p}{q} \in(0,1)$. We proceed by induction on $q$. The case $q=2$ is trivial. Assume validity for all values less than $q$. Let $q=h p+v$ be the Euclidean representation of $q$, such that $h$ and $v$ are nonnegative integers with $0 \leq v<p$. Let $A=10^{h-1}$ and $a=10^{h}$. We will proceed through several lemmas.

Lemma 1. If an equivalence class $[X]$ does not contain a representative formed by concatenating $A$ 's and a's, then $[X]$ is not admissible.

Proof. First let us note that we can choose a representative $X$ such that $S_{X}$ starts with 1 , and can therefore be written $S_{X}=10^{r_{1}} 10^{r_{2}} \ldots 10^{r_{p}}$, where $r_{1}, \ldots, r_{p}$ are nonnegative integers(which may be 0 ). We are therefore trying to prove that $r_{j}=h-1$ or $h$ for all $j$. Notice that if $r_{j}<h$ for all $j$, the string would have length less than or equal to $h p$ which is less than $q$, and if $r_{j} \geq h$ for all $j$, the string would have length greater than or equal to $(h+1) p$ which is greater than $q$. This observation gives us a lower bound on $X_{\max }$ and an upper bound on $X_{\min }$, as follows. By rotating if necessary we can assume that $r_{1} \leq h-1$, so that $M\left(X_{\max }\right) \geq .10^{h-1} 10^{q-(h+1)} 10^{h-1} 10^{q-(h+1)} \ldots$ Similarly, we can rotate so that $r_{1} \geq h$, rotate one more place to obtain a string beginning with $h 0$ 's, and then $M\left(X_{\min }\right) \leq .0^{h} 1^{q-h} 0^{h} 1^{q-h} \ldots$. Notice that these bounds 
for $M\left(X_{\max }\right)$ and $M\left(X_{\min }\right)$ have a distance less than $1 / 2$ between them, but just barely. Now, suppose that there is some $r_{j}$ greater than or equal to $h+1$. Then in the same manner we can bound $M\left(X_{\min }\right) \leq .0^{h+1} 1^{q-(h+1)} 0^{h+1} 1^{q-(h+1)} \ldots$, which is no longer within $1 / 2$ of the lower bound for $M\left(X_{\max }\right)$, and $[X]$ is therefore not admissible. Likewise, if there is some $r_{j}$ less than $h-1$, we obtain $M\left(X_{\max }\right) \geq$ $.10^{h-2} 10^{q-h} 10^{h-2} 10^{q-h} \ldots$, which is no longer within $1 / 2$ of the bound for $M\left(X_{\min }\right)$, and $[X]$ is not admissible. Thus, $r_{j}=h$ or $h-1$ for all $j$.

We see from this that we need only consider equivalence classes which contain an element formed by concatenating $A$ 's and $a$ 's. Suppose $[X]$ is such an equivalence class. $S_{X_{\max }}$ must begin with a 1 , and so can be written as $S_{X_{\max }}=Y_{1} Y_{2} \ldots Y_{p}$, where each of the $Y_{j}$ 's is either $A$ or $a$. On the other hand, $S_{X_{\min }}$ must begin with a 0 . To express $X_{\min }$ in a similar fashion let $A^{c}=0^{h-1} 1$ and let $a^{c}=0^{h} 1$. If $X_{\min }$ ended in a 0 , then we would have $D\left(M\left(X_{\min }\right)\right)<M\left(X_{\min }\right)$, which contradicts the choice of $X_{\min }$ as the minimal representative. Thus, $S_{X_{\min }}$ ends in a 1 , and can therefore be expressed as a concatenation of $A^{c}$ s and $a^{c}$ s. It is evident that we need to be able to determine how the distance function behaves on strings in the form of $S_{X_{\max }}$ and $S_{X_{\text {min }}}$. If $Y_{j}=A($ resp. $a)$ we will use the notation $Y_{j}^{c}$ to denote $A^{c}\left(\right.$ resp. $\left.a^{c}\right)$.

Lemma 2. Suppose that $S_{Y}=Y_{1} Y_{2} \ldots Y_{p}$, where each of the $Y_{j}$ 's is either A or a, and $S_{Z}=Z_{1} Z_{2} \ldots Z_{p}$, where each of the $Z$ 's is either $A^{c}$ or $a^{c}$. Then $d(Y, Z)<1 / 2$ if and only if one of the following conditions holds:

i) $Y_{1}{ }^{c}=Z_{1}$.

ii) $Y_{1}=a, Z_{1}=A^{c}$.

iii) $Y_{1}=A, Z_{1}=a^{c}$, and $Y_{j}=a, Z_{j}=A^{c}$, where $j$ is the smallest index greater than 1 for which $Y_{j}^{c} \neq Z_{j}$.

Proof. Since $M(Y)>M(Z), d(Y, Z)<1 / 2$ if, and only if, $M(Z)>M(Y)-1 / 2$. Suppose that $Y$ and $Z$ satisfy $($ iii $)$, so $S_{Y}=10^{h-1} Y_{2} \ldots Y_{p}, S_{Z}=0^{h} 1 Z_{2} \ldots Z_{p}$. Now, $Y_{2} \ldots Y_{p}$ can be written as $1 Y_{2}^{c} \ldots Y_{p-1}^{c} 0^{r}$, where $r$ is either $h$ or $h-1$. We are therefore comparing $10^{h-1} 1 Y_{2}^{c} \ldots Y_{p-1}^{c} 0^{r}$ with $0^{h} 1 Z_{2} \ldots Z_{p}$. Note that $M\left(10^{h-1} 1 Y_{2}^{c} \ldots Y_{p-1}^{c} 0^{r}\right)-$ $1 / 2=M\left(0^{h} 1 Y_{2}^{c} \ldots Y_{p-1}^{c} 0^{r}\right)$. Since $Y_{j}=a^{c}$ and $Z_{j}=A^{c}$, we see that $M\left(0^{h} 1 Y_{2}^{c} \ldots\right.$ $\left.Y_{p-1}^{c} 0^{r}\right)<M\left(0^{h} 1 Z_{2} \ldots Z_{p}\right)$, so that $d(Y, Z)<1 / 2$. That $(i)$ and $(i i)$ imply $d(Y, Z)<$ $1 / 2$ is similar, but simpler. The only other case to consider is when $Y_{1}=A, Z_{1}=a^{c}$, and $Y_{j}=A$ and $Z_{j}=a^{c}$, where $j$ is the smallest index greater than 1 for which $Y_{j}^{c} \neq Z_{j}$. The same argument shows that in this case $d(Y, Z)>1 / 2$. Note that if $Y_{1}^{c} \neq Z_{1}$, then there must be a $j>1$ such that $Y_{j}^{c} \neq Z_{j}$, since the number of $A$ 's present in $Y$ must equal the number of $A^{c}$ 's in $Z$, due to the fact that $Y$ and $Z$ have the same length. We have therefore exhausted all cases. This completes the proof of Lemma 2.

There is a very convenient symmetry to Lemma 2 which is based on the fact that Lemma 2 is essentially a statement about binary strings on symbols, one of which, $A$, is greater than the other, $a$. The statement of the theorem holds if we replace every $A$ or $A^{c}$ with a 1 and every $a$ or $a^{c}$ with a 0 . Let us isolate this as a new lemma, the proof of which we omit due to the fact that it is essentially identical to that of Lemma 2.

Lemma 3. Suppose that $Y=Y_{1} Y_{2} \ldots Y_{p}$, where each of the $Y_{j}$ 's is either 1 or 0 , and $Z=Z_{1} Z_{2} \ldots Z_{p}$, where each of the $Z$ 's is either 1 or 0 . Suppose that 
$M(Y)>M(Z)$. Then $d(Y, Z)<1 / 2$ if and only if one of the following conditions holds:

i) $Y_{1}=Z_{1}$.

ii) $Y_{1}=1, Z_{1}=0, Y_{j}=0$ and $Z_{j}=1$, where $j$ is the smallest index greater than 1 for which $Y_{j} \neq Z_{j}$.

We are now ready to complete the proof of Theorem 1 . We can apply the induction hypothesis to obtain a unique admissible equivalence class $[W]$ in $C_{p}^{v}$. Let $W=W_{1} \ldots W_{p}$, where each $W_{j}$ is either 1 or 0 . Let $[X]$ be the equivalence class in $C_{q}^{p}$ containing the element $X=W_{1}^{\prime} \ldots W_{p}^{\prime}$, where each $W_{j}^{\prime}=A$ if $W_{j}=1$ and $W_{j}^{\prime}=a$ if $W_{j}=0$. The claim now is that $[X]$ is the unique admissible equivalence class in $C_{q}^{p}$. Let $W_{\max }=Y_{1} \ldots Y_{p}, W_{\min }=Z_{1} \ldots Z_{p}$, where each of the $Y_{j}$ 's and $Z_{j}$ 's is either 1 or 0 . Then it follows that $X_{\max }=Y_{1}^{\prime} \ldots Y_{p}^{\prime}$ and $X_{\min }=\left(Z^{\prime}\right)_{1}^{c} \ldots\left(Z^{\prime}\right)_{p}^{c}$. Comparing Lemma 2 and Lemma 3 we see that the fact that $d\left(W_{\max }, W_{\min }\right)<1 / 2$ implies that $d\left(X_{\max }, X_{\min }\right)<1 / 2$. Uniqueness and statement (ii) regarding converses and palindromes all follow from the corresponding properties of the admissible element in $C_{p}^{v}$, and therefore $C_{q}^{p}$ contains a unique equivalence class with the required properties. This completes the induction. Statement (iii) of Theorem 3 is a simple consequence of uniqueness: if $\left[X_{T}\right]$ is admissible in $C_{q}^{p}$, then letting $S=T^{n}$ gives an admissible class $\left[X_{S}\right] \in C_{n q}^{n p}$, which is unique by the prior argument. This completes the proof of Theorems 2 and 3.

Acknowledgements. We'd like to thank Linda Keen for many helpful discussions. The third author is grateful for support from Australian Research Council grants DE140101201 and DP140100559.

\section{References}

[1] Ashley, J.: Marker automorphisms of the one-sided d-shift. - Ergodic Theory Dynam. Systems 10, 1990, 247-262.

[2] Blanchard, P., R. Devaney, and L. Keen: Complex dynamics and symbolic dynamics. - In: Symbolic dynamics and its applications, edited by S. G. Williams, Proc. Sympos. Appl. Math. 60, 2004, 37-60.

[3] Branner, B.: The Mandelbrot set. - In: Chaos and fractals: The mathematics behind the computer graphics, edited by R. Devaney and L. Keen, Proc. Sympos. Appl. Math. 39, 1988, 75-106.

[4] Bullet, S., and P. Sentenac: Ordered orbits of the shift, square roots, and the devil's staircase. - Math. Proc. Cambridge Philos. Soc. 115, 1994, 451-481.

[5] Carleson, L., and T. W. Gamelin: Complex dynamics. - Springer-Verlag, New York, 1995.

[6] FLEK, R.: On the dynamics of quasi-self-matings of generalized starlike complex quadratics and the structure of the mated Julia sets. - C.U.N.Y. Thesis, 2009.

[7] Goldberg, L. R.: Fixed points of polynomial maps. I. Rotation subsets of the circle. - Ann. Sci. Éc. Norm. Supér. 25, 1992, 679-685.

[8] Goldberg, L. R., and J. Milnor: Fixed points of polynomial maps. Part II. Fixed point portraits. - Ann. Sci. Éc. Norm. Supér. 26, 1993, 51-98.

[9] Keen, L.: Julia sets. - In: Chaos and fractals: The mathematics behind the computer graphics, edited by R. Devaney and L. Keen, Proc. Sympos. Appl. Math. 39, 1988, 57-73. 